\title{
Effect of BMP2-Platelet-rich Plasma-Biphasic Calcium Phosphate Scaffold on Accelerated Osteogenesis in Mastoid Obliteration
}

\author{
CHUL HO JANG ${ }^{1}$, CHEOL HEE $\mathrm{CHOI}^{2}$ and YONG BEOM CHO ${ }^{1}$ \\ ${ }^{1}$ Department of Otolaryngology, Chonnam National University Medical School, Gwangju, Republic of Korea; \\ ${ }^{2}$ Department of Pharmacolocy, Chosun Medical School, Gwangju, Republic of Korea
}

\begin{abstract}
The aim of this study was to evaluate the synergistic effect of platelet-rich plasma (PRP) and recombinant human bone morphogenic protein (BMP)-2 on accelerated osteogenesis of hydroxyapatite/ $\beta$-tricalcium phosphate mixture and biphasic calcium phosphate $(B C P)$ in mastoid obliteration. To the best of our knowledge, there have been no studies reporting the enhancing effects of $B C P$, combined with BMP2 and PRP, on osteogenesis in mastoid obliteration. Mastoid obliteration was performed in a control group (BCP only, $n=7$ ), a group treated with BMP 2 and $B C P$ (experimental group $I, n=7$ ), and a group treated with $B M P 2$, $P R P$ and BCP (experimental group II, $n=7$ ). The animals were administered fluorescent bone labels for a qualitative evaluation of bone formation; oxytetracycline hydrochloride was administered at 2 weeks, calcein at 4 weeks, and alizarin red at 8 weeks. The animals were sacrificed 12 weeks postsurgery and osteogenesis was evaluated by micro-computed tomography, histological investigation, and histomorphometry. Both experimental groups showed accelerated osteogenesis compared to the control group. However, there were no statistically significant differences between experimental groups I and II. From these results, it can be concluded that BMP2 activated BCP for the enhancement of bone regeneration. However, no synergistic effect of $B M P 2$ and PRP on the osteogenesis of BCP was observed.
\end{abstract}

The successful surgical eradication of mastoiditis usually requires removal of both diseased and sometimes normal

Correspondence to: Chul Ho Jang, MD, Ph.D., Department of Otolaryngology, Chonnam National University Hospital, Hakdong 8, Dongku, Gwangju, 501, South Korea. Tel: +82622206774 , email: chulsavio@hanmail.net

Key Words: Biphasic calcium phosphate, bone morphogenic protein-2, platelet-rich plasma. anatomic structures by an otolaryngologist. For example, a canal wall-down mastoidectomy is one common surgical technique, but it has variable long-term outcomes. The purpose of mastoid obliteration is to replace the open mastoid cavity with material that enhances osteogenesis so it can be free of infection and cholesteatoma. This leads to an epithelized lateral surface that isolates the mucosalized mesotympanic space while ablating the mucosalized and pneumatized mastoid space. Various materials, both biological and alloplastic, have been used in mastoid obliteration (1-6). Allografts, xenografts, or alloplastic bone substitutes are currently employed in clinics because autografts can lead to donor site morbidity and may lack osteogenic potential $(2,3,5,6)$. In particular, alloplastic bone substitutes, such as hydroxyapatite (HA) and $\beta$ tricalcium phosphate $(\beta$-TCP) ceramics, are very promising and avoid the disadvantage of autografts because their geometry and degradation properties can be adapted to the local demands; furthermore, transmission of infection can be avoided (7).

Biphasic calcium phosphate $(\mathrm{BCP})$ consists of a mixture of the more stable HA and the more soluble $\beta$-TCP, with varying $\mathrm{HA} / \beta$-TCP ratios (8). BCP has shown excellent biocompatibility; however, it cannot induce differentiation of mesenchymal stem cells into osteoblasts, which is required for mineralization and regeneration of bone tissue (9). For better osteogenesis of alloplastic bony scaffolds, then need for additional growth factors is gaining increasing attention. Bone morphogenic protein (BMP)-2 has been commonly used for osteoinduction using alloplastic scaffolds (10). BMP2 is a member of the BMP subgroup of the transforming growth factor (TGF) superfamily of proteins (11). It is generally accepted that additional use of BMP2 with alloplastic bony scaffolds accelerated osteogenesis in defective bones. We also observed accelerated osteogenesis of BMP2-coated bone scaffold in mastoid obliteration $(11,12)$. 
Platelet-rich plasma (PRP) contains proteins and growth factors that can promote differentiation of cells, and it was postulated that PRP may enhance healing at sites of injury by enhancing tissue regrowth and repair (13). The application of PRP gave successful results and beneficial outcomes in bone repair (14). The bone regenerative effect of PRP is known to be modulated by growth factors such as platelet-derived growth factor, insulin growth factor, and TGF $\beta$ (15-17).

As PRP stimulates osteoblastic differentiation in the presence of BMPs (18), it is assumed that PRP and BMP2 can act synergistically to enhance osteogenesis of alloplastic scaffold. However, to date, only three studies have demonstrated the efficacy of PRP along with BMP2 in bone regeneration (19-21). There has been considerable disagreement regarding the synergistic effect of PRP with BMP2. In the calvarial bony defect model, Yoshida et al. (19) and Jeon et al. (20) reported a positive effect, but Lim et al. (21) found no synergistic effect. The purpose of the present study was to determine whether the combination of alloplastic BCP with PRP and BMP2 can accelerate osteogenesis synergistically in mastoid obliteration.

\section{Materials and Methods}

Materials. Sprague-Dawley (SD) rats (male) were purchased from Samtako (Samtako Bio Korea Co., Osan, Korea). BCP and BCP coated with BMP2 (BMP2/BCP powder, $1 \mathrm{mg} / \mathrm{g}$ ) was obtained from CowellMedi (CowellMedi Co. Ltd, Pusan, Korea). Calcein blue, alizarin red was purchased from Sigma (Sigma Aldrich Co., Seoul, Korea), and oxytetracycline was purchased from Pfizer (Pfizer Korea, Seoul, Korea).

Preparation of platelet-rich plasma. The platelet-rich plasma was prepared based on a method previously described by Pantou et al. (22). Three rats were employed as PRP donors. Whole blood was collected by cardiac puncture and centrifuged at $220 \times g$ for $10 \mathrm{~min}$ to separate the red blood cell fraction from the whole plasma. A second centrifugation was carried out at $330 \times g$ for $15 \mathrm{~min}$ to separate PRP from platelet-poor plasma. PRP coagulated immediately with the BCP particles.

Surgery. Twenty-one male SD rats (age 12 weeks), with normal eardrums and Preyer reflexes, were used for the experiment and they were housed separately in sterile cages. All animal experiments followed a protocol approved by the Committee for Animal Experimentation at Chonnam National University, Korea (CNU IACUC-H-2014-16). The animals were divided into three groups. The rats were anesthetized with an intraperitoneal injection of zoletil and xylazine. Lidocaine (1\%), with $1 / 100,000$ epinephrine,was injected into the soft tissue over the tympanic bulla and then a postauricular incision was made. The tympanic bulla was exposed and a round hole was created by drilling. After removing the mucoperiosteum of the bulla by a microelevator with alligator forceps, bulla obliteration was performed using BCP in the control group ( $n=7)$, BMP2 with BCP in experimental group I $(n=7)$, and BMP2 with PRP and BCP in experimental group II $(n=7)$. This study

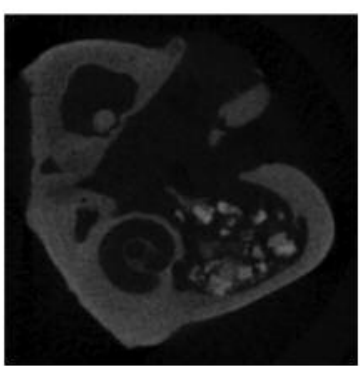

Control

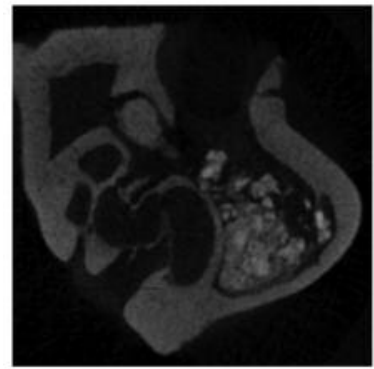

Experimental group I

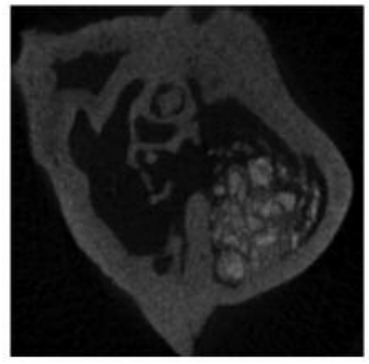

Experimental group II
Figure 1. Microcomputed tomographic analysis showed more effective osteogenesis at 8 weeks in both experimental groups compared to the control group. However, there were no significant differences between the experimental groups.

used BMP2 at $1 \mathrm{mg} / \mathrm{g}$ and PRP at $1 \mathrm{mg} / \mathrm{g} \mathrm{BCP}$ powder. The bony hole of the bulla was covered with compressed gelfoam. The wounds were then closed. Ciprofloxacin was injected intramuscularly for prevention of infection.

Fluorescent bone labeling. The rats were administered fluorescent bone labels for the qualitative evaluation of sequential bone formation. In order to assess the active mineralization of new bone formation, each group received oxytetracycline hydrochloride intravenously at 4 weeks, calcein blue at 8 weeks, and alizarin red at 10 weeks (Sigma-Aldrich Chemical Co.).

Ex vivo bulla micro-computed tomography (micro-CT). The animals of each group were sacrificed 12 weeks post-surgery. Ex vivo microCT images of osteogenesis were obtained.

Histological evaluation. The bullae were harvested from all the rats. They were fixed in a formaldehyde solution (10, \% for $48 \mathrm{~h}$ ). Samples were then embedded in Technovit 7200 VLC (Kulzer \& Co. GmbH, Wehrhein, Germany), a glycol methacrylate solution. After polymerization, the samples were processed using the sawing and grinding technique. After examination of the fluorescent labels by confocal microscopy (Leica, Wetzlar, Germany), the specimens were stained with hematoxylin and eosin. Histomorphometric analysis of new bone formation in the middle ear of each group was evaluated by a PC-based image analysis system (Image Inside, Focus Technology, Daejon, Korea). One-way ANOVA test was employed for statistical analysis. The statistical significance was set at $5 \%$ and $p$-values were adjusted for multiple comparisons. 


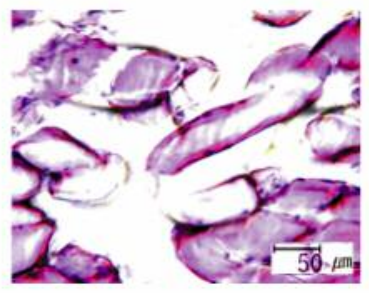

Control group

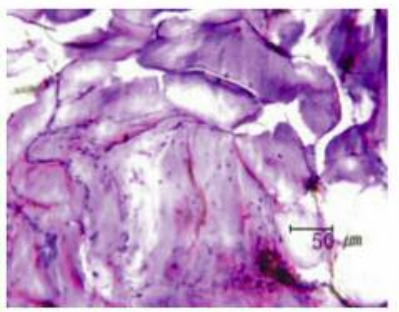

Experimental group I

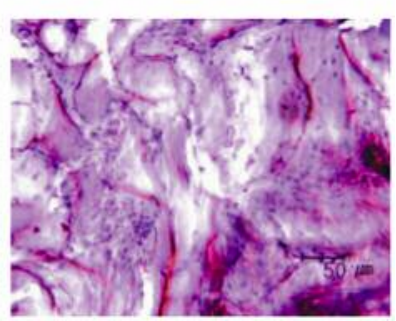

Experimental group II
Figure 2. Histological assessment at 8 weeks showed more prominent osteogenesis in both the experimental groups than in the control group. Experimental group II showed more enhanced osteogenesis as compared to that in experimental group I.

\section{Results}

Micro-CT analysis. Experimental groups showed enhanced osteogenesis compared to that shown by the control group. The interspace between each BCP granule was closed by new bone formation in the experimental groups. Bone formation observed at the bulla cavity margin in experimental groups I and II was accelerated compared to that observed in the control group (Figure 1).

Histological assessment by light microscopy. Neither experimental nor control groups exhibited signs of resorption of BCP particles at 12 weeks of obliteration as observed by light microscopy. Limited osteogenesis between BCP particles was seen in the control group. However, in experimental groups I and II, BCP particles were embedded in dense cellular connective tissue, which indicates prominently enhanced osteogenesis. Experimental group II showed more mildly enhanced osteoinduction as compared to experimental group I (Figure 2). However, histomorphometric findings showed no significant difference in enhancement of osteogenesis between experimental groups I and II (Figure 3).

Confocal microscopic analysis. The deposition of calcium ions, which corresponded to bone formation, was visible as the presence of small amounts of green, blue, and red colors. The different colored fluorescent dyes allowed for a description of the time course of new bone formation. This

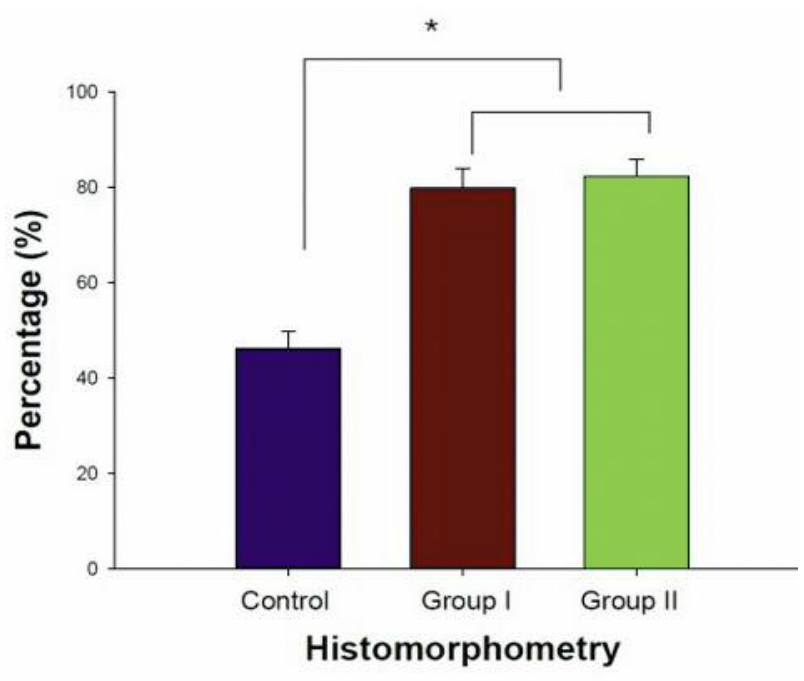

Figure 3. Histomorphometric findings of new bone formation showed significantly enhanced osteogenesis in both experimental groups than in the control group. However, there were no significant differences between the experimental groups; ${ }^{*} p<0.05$.

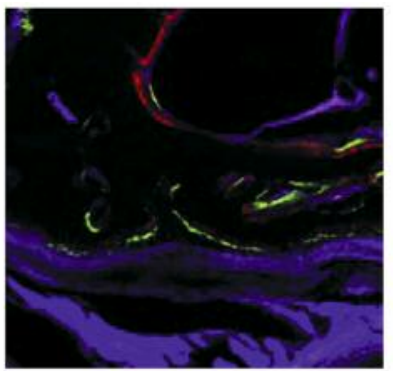

Control group

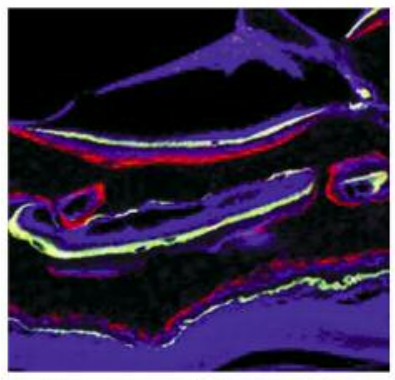

Experimental group I

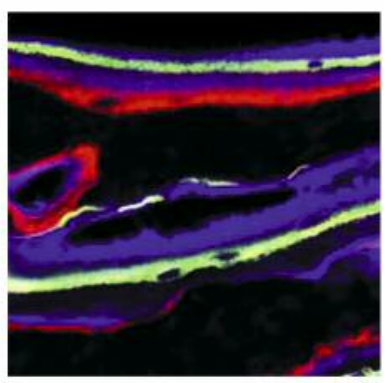

Experimental group II
Figure 4. Confocal microscopy showed more enhanced intensity in both experimental groups than in the control group at 2,4, and 8 weeks. However, there were no significant differences between the experimental groups.

characterization revealed a small amount of calcium deposition, at 2, 4, and 8 weeks after obliteration. More intense and homogenous bands of integrated fluorochromes were observed at 2, 4 and 8 weeks in experimental groups I 
and II than in the control group. However, there were no significant differences between experimental groups I and II (Figure 4).

\section{Discussion}

The use of autogenous bone pate remains the gold standard for bone repair involving mastoid obliteration. In the case of mastoid obliteration, it is difficult to collect sufficient bone pate in revision surgery. Many synthetic bone scaffolds have been used as bone substitutes in mastoid obliteration. Regarding the acceleration of bone formation on BCP ceramics, both HA and TCP are well known for functioning as carriers of BMP2(18). Previous studies have shown that the incorporation of BMP2 into bone scaffolds accelerates bone formation in mastoid obliteration $(10,11)$. The capacity of PRP to stimulate bone regeneration is generally considered to be through stimulatory effects of growth factors released from activated platelets on progenitor cells and vascularization at local sites $(20,23)$.

In the present study, both experimental groups I and II demonstrated greater osteogenesis as compared to the control group. However, there was no statistically significant difference in osteogenesis between groups I and II. The exact effect of PRP on osteogenesis remains to be elucidated. Our results are different from those of Yoshida et al. (19) and Jeon et al. (20). We believe that osteogenesis in calvarial defect using bony scaffold is different from that in mastoid obliteration. For mastoid obliteration, mastoid mucosa should be excised. In calvarial bony defect (19-21), the pericranium, which is periosteum of the skull bones, has a potential role in regeneration due to having an excellent blood supply. Additionally, the pericranium provides a niche for pluripotent cells and a source of molecular factors (24). Moreover, the dura is more osteogenic than the pericranium (25). Another probable explanation may be the nature of the scaffold, which is autogenous or alloplastic. Additional use of PRP in autogenous bone scaffold stimulates osteogenesis by releasing several growth factors contained in PRP stimulating angiogenesis and the proliferation of osteoprogenitor cells only in the early period after transplantation; conversely, PRP has no positive effect on osteogenesis when used with alloplastic or xenogenic scaffolds (26). Moreover, PRP highly varies from donor to donor, and each PRP preparation can differ in its concentration of proteins and growth factors (27). To the best of our knowledge, there have been no reports on the synergistic effects of bone scaffold combined with BMP2 and PRP on osteogenesis in mastoid obliteration. From these results, it can be concluded that BMP2-activated BCP can enhance bone regeneration. However, there was no synergistic effect of BMP2 and PRP on osteogenesis of BCP. Further studies are required to elucidate the clinical applications of PRP.

\section{Acknowledgements}

This study was supported by a grant (CRI 14 022-1) Chonnam National University Hospital Biomedical Research Institute and supported by Fishery Commercialization Technology Development Program, Ministry for Food, Agriculture, Forestry and Fisheries, Republic of Korea (grant no. A120942).

\section{References}

1 Moffat DA, Gray RF and Irving RM: Mastoid obliteration using bone pate. Clin Otol 19: 149-157, 1994.

2 Yung $M$ and Smith P: Mid-temporal pericranial and inferiorly based periosteal flaps in mastoid obliteration. Otolaryngol Head Neck Surg 137: 906-912, 2007.

3 Matsuno T, Nakamura T, Kuremoto K, Notazawa S, Nakahara $\mathrm{T}$, Hashimoto Y, Satoh T and Shimizu Y: Development of $\beta$ tricalcium phosphate/collagen sponge composite for bone tissue regeneration. Dent Mater J 25: 138-144, 2006.

4 Jang $\mathrm{CH}$, Park H, Cho YB and Song CH: Mastoid obliteration using a hyaluronic acid gel to deliver a mesenchymal stem cellsloaded demineralized bone matrix: an experimental study. Int $\mathrm{J}$ Pediatr Otorhinolaryngol 72: 1627-1632, 2008.

5 Jang $\mathrm{CH}$, Cho YB and Bae CS: Evaluation of bioactive glass for mastoid obliteration: a guinea pig model. In Vivo 21: 651-655, 2007.

6 Yanagihara N, Komori M and Hinohira Y: Total mastoid obliteration in staged canal-up tympanoplasty for cholesteatoma facilitates tympanic aeration. Otol Neurotol 30: 766-770, 2009.

7 Eppley BL, Pietrzak WS and Blanton MW: Allograft and alloplastic bone substitutes: a review of science and technology for the craniomaxillofacial surgeon. J Craniofacial Surg 16: 981-989, 2005.

8 Lim HC, Hong JY, Lee JS, Jung UW and Choi SH: Late-term healing in an augmented sinus with different ratios of biphasic calcium phosphate: a pilot study using a rabbit sinus model. J Periodontal Implant Sci 46: 57-69, 2016.

9 Escobar T, Almeida e Sousa J, Portela A, Vasconcelos M and Faria de Almeida R: The effect of a biphasic calcium phosphate on bone healing: a pilot study in rats.Int $\mathrm{J}$ Oral Maxillofac Implants 29: 1322-1331, 2014.

10 Alam MI, Asahina I, Ohmamiuda K, Takahashi K, Yokota S and Enomoto S: Evaluation of ceramics composed of different hydroxyapatite to tricalcium phosphate ratios as carriers for rhBMP2. Biomaterials 22: 1643-1651, 2001.

11 Jang CH, Kim MS, Cho YB, Jang YS and Kim GH: Mastoid obliteration using 3D PCL scaffold in combination with alginate and rhBMP2. Int J Biol Macromol 62: 614-622, 2013.

$12 \mathrm{Kim}$ BS, Choi MK, Yoon JH and Lee J: Evaluation of bone regeneration with biphasic calcium phosphate substitute implanted with bone morphogenetic protein 2 and mesenchymal stem cells in a rabbit calvarial defect model. Oral Surg Oral Med Oral Pathol Oral Radiol 120: 2-9, 2015.

13 Marx RE: Platelet-rich plsasma (PRP): What is PRP and what is not PRP? Implant Dent 10: 225-228, 2001.

14 Tözüm TF and Demiralp B: Platelet-rich plasma: a promising innovation in dentistry. J Can Dent Assoc 69: 664, 2003.

15 Simman R, Hoffmann A and Bohinc RJ: Role of platelet-rich plasma in acceleration of bone fracture healing. Ann Plast Surg 61: 337-344, 2008. 
16 Hokugo A, Sawada Y, Hokugo R, Iwamura H, Kobuchi M, Kambara T, Morita $\mathrm{S}$ and Tabata Y: Augmented bone regeneration activity of platelet-rich plasma by biodegradable gelatin hydrogel. Tissue Eng 11: 1224-1233, 2005.

17 Fekkag BH, Delorme B, Sohier J, Magne D, Hardouin P and Layrolle P: Macrophage and osteoblast responses to biphasic calcium phosphate microparticles. J Biomed Mater Res Part A 93: 1588-1595, 2010.

18 Tomoyasu A, Higashio K, Kanomata K, Goto M, Kodaira K, Serizawa H, Suda T, Nakmura A, Nojima J, Fukuda T and Katagiri T: Platelet-rich plasma stimulates osteoblastic differentiation in the presence of BMPs.Biochem Biophys Res Commun 361: 62-67, 2007.

19 Yoshida K, Sumita Y, Marukawa E, Harashima M and Asahina I: Effect of platelet-rich plasma on bone engineering with an alloplastic substitute containing BMP2. Biomed Mater Eng 23: 163-172, 2013.

20 Jeon YR, Jung BK, Roh TS, Kang EH, Lee WJ, Rah DK, Lew DH and Yun IS: Comparing the effect of nonactivated plateletrich plasma, activated platelet-rich plasma, and bone morphogenetic protein-2 on calvarial bone regeneration. J Craniofac Surg 27: 317-321, 2016.

21 Lim HP, Mercado-Pagan AE, Yun KD, Kang SS, Choi TH, Bishop J, Koh JT, Maloney W, Lee KM, Yang YB and Park SW: The effect of rhBMP2 and PRP delivery by biodegradable $\beta$ tricalcium phosphate scaffolds on new bone formation in a nonthrough rabbit cranial defect model. J Mater Sci Mater Med 24: 1895-1903, 2013.
22 Pantou AL, Markopoulou CE, Dereka XE, Vavouraki H, Mamalis A and Vrotsos IA: The effect of platelet-rich plasma (PRP) combined with a bone allograft on human periodontal ligament (PDL) cells. Cell Tissue Bank 13: 81-88, 2012.

23 Boyapati L and Wang HL: The role of platelet-rich plasma in sinus augmentation: a critical review. Implant Dentistry 15: 160$170,2006$.

24 Ferretti C and Mattioli-Belmonte M: Periosteum derived stem cells for regenerative medicine proposals: Boosting current knowledge. World J Stem Cells 6: 266-277, 2014.

25 Gosain AK, Santoro TD, Song LS, Capel CC, Sudhakar PV and Matloub HS: Osteogenesis in calvarial defects: contribution of the dura, the pericranium, and the surrounding bone in adult versus infant animals. Plast Reconstr Surg 112: 515-527, 2003.

26 Jung RE, Schmoekel HG, Zwahlen R, Kokovic V, Hammerle CH and Weber FE: Platelet-rich plasma and fibrin as delivery systems for recombinant human bone morphogenic protein-2. Clin Oral Implant Res 16: 676-682, 2005.

27 Ranly DM, Lohmann CH, Andreacchio D, Boyan BD and Schwartz Z: Platelet-rich plasma inhibits demineralized bone matrix-induced bone formation in nude mice. J Bone Joint Surg Am 89: 139-147, 2007.
Received July 29, 2016

Revised August 24, 2016

Accepted September 5, 2016 